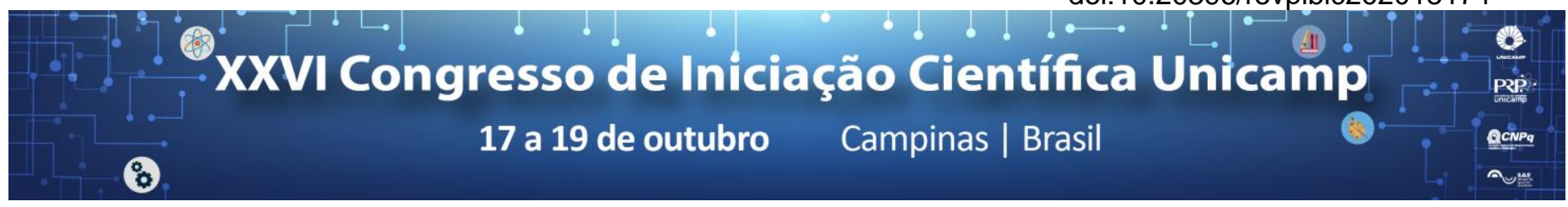

\title{
A Study on the Minimum Subgraph Diameter Problem
}

\author{
Arthur Pratti Dadalto*, Fábio Luiz Usberti, Mário César San Felice
}

\begin{abstract}
This work addresses the minimum subgraph diameter problem (MSDP), an NP-hard problem. Given a graph with lengths and costs associated with its edges, the MSDP consists in finding a spanning subgraph with total cost limited by a given budget, such that its diameter is minimum. We prove that there is no $\beta$-approximation algorithm for the MSDP, for any constant $\beta$ unless $P=N P$. Moreover, we propose a benchmark of instances and present a computational study of mixed integer linear programming formulations and genetic algorithms for the MSDP.
\end{abstract}

\section{Key words:}

Approximation algorithm, integer linear programming, computational study.

\section{Introduction}

In a combinatorial optimization problem, there is a set of instances, a finite set of feasible solutions for each instance and an objective function. A feasible solution is one that respects a set of restrictions associated with the problem. In a minimization problem, the goal is to find a feasible solution such that its value, evaluated by the objective function, is minimum. Such a solution is called optimal.

We study the minimum subgraph diameter problem (MSDP). Given a graph with lengths and costs associated with its edges, the MSDP consists in finding a spanning subgraph with total cost limited by a given budget, such that its diameter is minimum.

Plesnik [1] first presented this problem and proved that for any $\beta<2$, finding a $\beta$-approximation for the MSDP is NP-hard. Therefore, the MSDP itselft is also NP-hard.

The MSDP can appear in real world applications, such as the construction of a telecommunication network with a budget constraint. There are costs related to building connections, e.g. acquisition of cables, and there are transmission delays between nodes inherent to the network topology. A relevant goal could be to minimize the largest delay between two nodes in the resulting network.

This work aims to apply exact and metaheuristic optimization methods to the MSDP, as well as investigate the possibility of approximation algorithms for it.

\section{Results and Discussion}

Based on a theorem by Bálint [2] and a reduction from the problem explored in his paper to a $\beta$-approximation for the MSDP, we prove that there is no polynomial time $\beta$ approximation algorithm for the MSDP for any constant $\beta$, unless $P=N P$.

We propose two mixed integer linear programming (MILP) models for the MSDP. The first uses $\Theta(E)$ integer variables and $\Theta\left(V^{2} E\right)$ continuous variables. The second uses $\Theta(V E)$ integer only variables and is slower in general but could be useful for larger instances as the first uses $\Theta(V)$ times more memory.
For this first MILP model, we were also able to implement lazy constraints. This reduces the initial size of the model and adds constraints only as they become necessary using a separation algorithm, improving performance.

We present valid inequalities for both MILP models based on cover inequalities and tree connectivity inequalities. The inequalities found are specific to the MSDP, however stronger than the general ones applied by MILP solvers.

A metaheuristic solution for the MSDP was developed by using a Biased Random Key Genetic Algorithm (BRKGA) framework, a standardized method of applying genetic algorithms towards a problem. To use a BRKGA for a specific problem, it is only necessary to define a decoding algorithm from the chromosome to a solution for the problem and evaluate the chromosome fitness. The BRKGA API used was made by Toso and Resende and presented on their paper [3].

We propose a benchmark of randomly generated instances for the MSDP. Using this benchmark, a computational study of all optimization methods explored is presented.

\section{Conclusions}

Our result on the approximability of the MSDP strengthens the previously published bound and cuts short any effort to find a $\beta$-approximation for the MSDP. Nonetheless, a promising line of research is to investigate $(\alpha, \beta)$-approximation algorithms for the MSDP.

This work aimed at building a strong base for futures studies on the MSDP with the proposed benchmark of instances as well as with the MILP formulations, valid inequalities and meta-heuristics.

Acknowledgement

This project is supported by CNPq grant 127953/2017-5.

${ }^{1}$ Plesnik, J. (1981), The complexity of designing a network with minimum diameter. Networks, 11: 77-85.

2 Bálint, V. (2003). The non-approximability of bicriteria network design problems. Journal of Discrete Algorithms, 1:339-355.

3 R. F. Toso and M. G. C. Resende. A c++ application programming interface for biased random-key genetic algorithms. AT\&T Labs Research Technical Report, 2011. 